\title{
Hall Effect in ZnO Extrinsic Structure
}

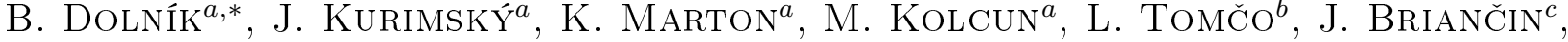 \\ M. FABiÁn ${ }^{c}$, M. HALAMA ${ }^{d}$, M. VOJTKO $^{d}$, M. RAJŇÁK ${ }^{e}$ \\ ${ }^{a}$ Department of Electrical Power Engineering, TU of Košice, Mäsiarska 74, 04120 Košice, Slovakia \\ ${ }^{b}$ Department of Aerodynamics and Simulations, TU of Košice, Rampová 7, 04121 Košice, Slovakia \\ ${ }^{c}$ Institute of Geotechnic, SAS, Watsonova 45, 04353 Košice, Slovakia \\ ${ }^{d}$ Department of Material Science, TU of Košice, Park Komenského 11, 04001 Košice, Slovakia \\ ${ }^{e}$ Institute of Experimental Physics SAS, Watsonova 47, 04001 Košice, Slovakia
}

\begin{abstract}
Zinc oxide-based extrinsic composite was investigated. The sample was selected from a series of components of one production batch, prepared by standard sintering technology. The content of extrinsic elements in $\mathrm{ZnO}$ base was determined by SEM. Van der Pauw method with four-point electrode fixture was used for study of conducting phenomena in square-shaped sample. It is normaly preferred to assume the symmetric uniformity of the electrical properties of sample, for which sheet resistance, bulk resistivity and Hall mobility, sheet carrier density and carrier concentration can be calculated. When the uniformity of measured parameters is breached, the anisotropy in the arrangement of the internal structure may be the cause. There remains the question of whether the extrinsic $\mathrm{ZnO}$ material can be isotropic, regarding the electrical conductivity. Although the Hall effect has been measured, preliminary measurements indicate the presence of anisotropy in the measured samples. Before measurement the following phenomena should be taken into account: magneto-electric effect, photo-electric effect and the isothermal condition should be preserved. Paper discusses the uniformity deviations for the defined setup configurations for positive and negative magnetic field directions. Bulk resistivity has been calculated by numerical solution of van der Pauw equation. Large offset voltage during the measurement is discussed.
\end{abstract}

DOI: 10.12693/APhysPolA.126.76

PACS: 61.43.Dq, 72.20.My, 84.32.Ff, 84.37.+q, 81.05.Xj, 72.80.Ng

\section{Introduction}

Zinc oxide $(\mathrm{ZnO})$ can be regarded as a material of the future, because it has unique electrical properties. These properties are of great interest for fundamental research, but also for the possible development of new technologies. Areas of application include many disciplines. At present, the possible areas of application include solar cells, gas sensors, ultraviolet (UV) photo-detectors, UV radiation source, transparent electronics, chemical absorbers, electrical and optical devices, catalysts for photocatalytic decomposition, piezoelectric sensors, electrostatic dissipative coating, biomedical science, and many others [1-3].

Electron diffraction data on $\mathrm{ZnO}$ first appeared in 1935 [4]. In 1960, good piezoelectric properties of $\mathrm{ZnO}$ were discovered. Various growth techniques of $\mathrm{ZnO}$ substrate are used, basics of which were developed during the 60th to 70th of the last century. Temperature dependent Hall measurements of $\mathrm{ZnO}$ were first reported in 1954, confirming the inherently N-type nature of this material [5]. Extremely nonlinear properties and the ability to absorb the energy of electronic components based on semiconductive ceramics brought a new idea that nanoceramics can be used as part of electric protection. $\mathrm{ZnO}$ has perhaps the most various types of nanostructured forms,

*corresponding author; e-mail: bystrik.dolnik@tuke.sk regarding both the structure and the properties. Understanding the role of native point defects (i.e. vacancies, interstitials, and antisites) and the integration of impurities is the key to control the conductivity of $\mathrm{ZnO}$. The intrinsic defect levels that lead to N-type doping lie approximately $0.05 \mathrm{eV}$ below the conduction band. While N-type $\mathrm{ZnO}$ is easily realized via excess of $\mathrm{Zn}$ or doping with $\mathrm{Al}, \mathrm{Ga}$, or In, the P-type doping has proven difficult to achieve.

The Hall mobility of electrons at $300 \mathrm{~K}$ for low N-type conductivity is $\mu_{H}=200 \mathrm{~cm}^{2} \mathrm{~V}^{-1} \mathrm{~s}^{-1}$, and for low P-type conductivity the hole mobility is $5-50 \mathrm{~cm}^{2} \mathrm{~V}^{-1} \mathrm{~s}^{-1}$. Free exciton binding energy amounts to $60 \mathrm{meV}$, which makes the excitons more stable at room temperature. There is possibility to fabricate heterostructures and to tune the band gap of $\mathrm{ZnO}$ from $3.3 \mathrm{eV}$ (at room temperature) to $4 \mathrm{eV}$ by alloying with $\mathrm{Mg}$ and to $2.9 \mathrm{eV}$ by making the $\mathrm{ZnCdO}$ alloys. $\mathrm{ZnO}$ ceramic varistors with $\mathrm{Bi}_{2} \mathrm{O}_{3}$ additives have been widely used to protect electronic circuits and power distribution lines from destructive overvoltages induced by lightning impulses or switching surges. The highly nonlinear current-voltage characteristics of these devices is due to the cumulative effect of many potential barriers at the grain boundaries of the polycrystalline $\mathrm{ZnO}$ material. $\mathrm{ZnO}$ based varistor is a very complex chemical system. Additives such as Bi, Pr, Mn, $\mathrm{Co}, \mathrm{Cu}, \mathrm{Sb}, \mathrm{V}, \mathrm{Cr}$ and $\mathrm{Al}$ together with the composition and the microstructure have important role in defining the electrical properties of $\mathrm{ZnO}$ based varistors. 


\section{Experimental procedure and results}

A set of identical $\mathrm{ZnO}$ extrinsic structure samples was tested. Van der Pauw method was used for Hall effect measurement. Square-shaped specimens with sides of $(9.96 \mathrm{~mm} \times 9.94 \mathrm{~mm})$ measured with accuracy of $0.01 \mathrm{~mm}$ and thickness of $0.667 \pm 0.004 \mathrm{~mm}$ were used. The study of the composition of the components in the samples by weight and atomic percent was made in SEM and the results are as follows (weight): O $14.27 \%$, Zn $74.37 \%$, Bi $4.54 \%$, Sb $3.83 \%$, Ni $0.71 \%$, Cr $0.62 \%$, Co $0.69 \%$, Mn $0.60 \%$ and Si $0.37 \%$. For study of conducting phenomena across the square shaped samples the van der Pauw method for resistivity measurement and Hall voltage measurement with four point electrode fixture was used. For a given applied dc current a set of 16 measurements was performed, consisting of a series of eight measurements for the resistivity calculation, followed by eight measurements of the Hall voltage. The first series was realized without the presence of a magnetic field, the second and third series were carried out in the presence of positively and negatively oriented static magnetic field.

Each individual measurement lasted for 600 seconds, in order to reduce the impact of initial polarization effects in the measured sample. Subsequent measurements followed after 30 minutes in order to minimize the effect of remaining space charge in the sample. To exclude the impact of the photoelectric effect (observed in the experiment) during the measurement, each sample was placed in a dark space. The experiments were done at room temperature. From the voltages measured in van der Pauw configuration the following parameters were calculated: sheet resistance $R_{S}$, bulk resistivity $\rho$, Hall mobility $\mu_{H}$, sheet carrier density $n_{S}$, carrier concentration $n$ and carrier type (P-type or N-type). The sheet resistance has been calculated by numerical solution of the van der Pauw formula [6]

$$
\exp \left(-\pi R_{12,34} / R_{S}\right)+\exp \left(-\pi R_{23,41} / R_{S}\right)=1,
$$

where $R_{S}$ is the sheet resistance, $R_{12,34}$ is resistance calculated from Ohm's law as $R_{12,34}=V_{34} / I_{12}, V_{34}$ is a DC voltage measured between contacts marked 3 and 4 on the sample with no externally applied magnetic field, $I_{12}$ is positive DC current injected into contact 1 and taken out of contact 2 of the sample. In a similar way the resistance $R_{23,41}=V_{41} / I_{23}$ was calculated. The bulk resistivity was calculated as $\rho=R_{S} \times d$, where $d$ is the thickness of the sample.

All of the above physical quantities, characterizing the conductivity phenomena in the sample were calculated and measured at different intensities of electric field. An overview of calculated physical parameters of the samples is shown in Table.

\section{Conclusions and discussion}

Analysis of physical quantities from Table leads to several conclusions. The sheet resistance slightly decreases with increasing voltage and is of the order of several $\mathrm{M} \Omega$.

Calculated physical quantities for $\mathrm{ZnO}$ extrinsic structure.

\begin{tabular}{c|c|c|c|c|c|c}
\hline \hline $\begin{array}{c}\text { Voltage } \\
\text { applied } \\
(\mathrm{V})\end{array}$ & $\begin{array}{c}\mathrm{R}_{S} \\
\times 10^{6} \\
(\Omega)\end{array}$ & $\begin{array}{c}\rho \\
\times 10^{5} \\
(\Omega \mathrm{cm})\end{array}$ & $\begin{array}{c}\mu_{H} \\
\left(\mathrm{~cm}^{2} \mathrm{~V}^{-1} \mathrm{~s}^{-1}\right)\end{array}$ & $\begin{array}{c}\mathrm{n}_{S} \\
\times 10^{7} \\
\left(\mathrm{~cm}^{-2}\right)\end{array}$ & $\begin{array}{c}\mathrm{n} \\
\times 10^{8} \\
\left(\mathrm{~cm}^{-3}\right)\end{array}$ & $\begin{array}{c}\text { Car- } \\
\text { rier } \\
\text { type }\end{array}$ \\
\hline 1100 & 3.69 & 2.46 & $1.92 \times 10^{4}$ & 8.84 & 13.3 & $\mathrm{~N}$ \\
\hline 1300 & 3.26 & 2.24 & $5.31 \times 10^{4}$ & 350 & 525 & $\mathrm{P}$ \\
\hline 1500 & 3.23 & 2.15 & $3.88 \times 10^{4}$ & 4.99 & 7.48 & $\mathrm{P}$
\end{tabular}

A similar trend has also the bulk resistivity, which is of the order of several hundreds of $\mathrm{k} \Omega$.

Regarding the mobility, the values are relatively large compared with the intrinsic $\mathrm{ZnO}$, except when measured at the applied voltage of $1300 \mathrm{~V}$. The sheet carrier density and carrier concentration varies by two orders of magnitude. An interesting result is obtained regarding the carrier type, whereas at lower electric field strength material appears to be of N-type, at higher electric fields strength it is of P-type.
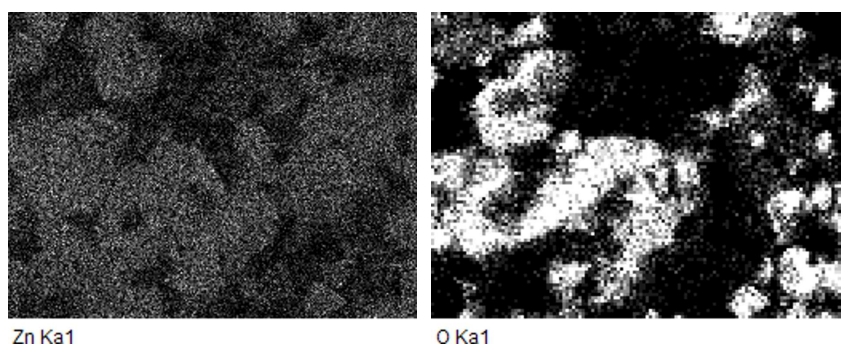

Fig. 1. Map of the distribution of elements.

It should be added that the measurements by the van der Pauw method point out on the non-uniformity of the sample's electrical properties. The authors believe that this is due to spatial anisotropy in the internal structure of the sample, which is confirmed by SEM study. A map of the distribution of elements (namely of zinc and oxygen) is shown in Fig. 1. During the measurements, it was found that $\mathrm{ZnO}$ extrinsic structure is sensitive to daylight. This phenomenon remains to be examined in detail in the future.

\section{Acknowledgments}

This work was supported by the projects VEGA No. 1/0388/13, VEGA No. 2/0045/13, VEGA No. $2 / 0043 / 12$.

\section{References}

[1] R. Hong, T. Pan, J. Qian, H. Li, Chem. Eng. J. 119, 71 (2006).

[2] J.H. Jun, H. Seong, K. Cho, B.-M. Moon, S. Kim, Ceramics International 35, 2797 (2009).

[3] M. Matsuoka, Jpn. J. Appl. Phys. 10, 736 (1971).

[4] H.J. Yearian, Phys. Rev. 48, 631 (1935).

[5] Z.Ch. Feng, Handbook of Zinc Oxide and Related Materials CRC Press, London, UK 2012.

[6] L.J. van der Pauw, Philips Tech. Rew. 20, 220 (2004). 